\title{
Theory of the Ultimate Bearing Capacity Calculation
}

\author{
Chang Yi Wang \\ Penglai City Land Resources Bureau, Shandong Province, China \\ Ben Jun Wang \\ Physics Institute of Ludong University, Shandong Province, China
}

Shu Zun Jiang

Modern Education Department of Ludong University, Shandong Province, China

Received: November 20, 2011 Accepted: December 6, $2011 \quad$ Published: February 1, 2012

doi:10.5539/esr.v1n1p109 URL: http://dx.doi.org/10.5539/esr.v1n1p109

\begin{abstract}
The traditional formula and calculation method for calculating the ultimate bearing capacity is not scientific. According to the research, the ultimate bearing capacity is equal to the real degree of the material and detection force product. Ultimate bearing capacity is not for the change of detecting the force. On the basis of this understanding, a new theory of the study bearing capacity of rock-soil and new methods is generated.
\end{abstract}

Keywords: Ultimate bearing capacity, Compaction characteristic value, Detecting force

\section{Introduction}

In geotechnical investigation, determine the bearing capacity of foundation soil constitutes an important task. The bearing capacity of a rock-soil is the important data of what the reconnaissance unit must provide to the project design unit and construction unit, the accuracy, reliability of the data relation to the construction safety and engineering investment of economic rationality. At present, there are many ways to determine the bearing capacity of foundation soil, but can be summed up mainly three kinds: 1) load test, in situ test; 2) formula; 3) experience judging. Among them, load test direct, accurate, but limited to the conditions it was not adopted generally. Formula, should also say, is accurate and reliable, but, so far, there is no the formula which access to a complete, unified, reflecting exactly the direct relation rule between the detection force and the bearing capacity. At present, the people are using the calculation formula of the bearing capacity, some to being from theoretical derivation, some from experience, but, in these formulas, there are many the parameters difficult to properly determine, and cannot reflect uniformly the actual relationship law between the bearing capacity and the detecting force. Therefore, at present, the formula calculation method exist still a scientific, accuracy problem. Because the physical properties of soil and the bearing capacity corresponding indicators in table was cancelled in the new national standard $<$ the standard for design of building foundation $>$ (GB50007-2002). what be issued, so, at present people pay more attention to the theoretical formula to calculate the bearing capacity method. However, there is no the formula of the direct relationship between bearing capacity and detecting force, so, in the current engineering practice, the method of what using the empirical formula roughly estimate is still the prevailing. Therefore, improve the theory of the relationship between the bearing capacity and the forces, establish a scientific calculation formula of the bearing capacity, still constitute an important subject in the engineering survey study.

In short, the traditional formula for calculating the ultimate bearing capacity and calculation method is not scientific. To change the current situation, we also need to further understand the relation law between the fundamental limit bearing capacity and detection force, establish a scientific and unified formula for calculating the ultimate bearing capacity. This paper conducted a thematic exploration, given a new theory of the relationship between the ultimate bearing capacity and the detection force, new method and new formula.

\section{The Direct Relationship between the Ultimate Bearing Capacity and the Force Detection}

According to research, the force is constituted by a real force and a false force. The formula between the real 
force $F_{T}$ and force $F$ is

$$
F_{T}=T F
$$

In this Type, $T$ is called the real degree, including close-grained degree, degree of hardness, complete degree, the degree of stability, not plastic degree, and immutable degree and so on. False force is equal to force by false degree. Namely,

$$
F_{F}=E F
$$

In this Type, $E$ is called the false degree of the object, including unreal degree, weak degree, inattentive degree, broken degree, porosity, plastic degree, variable degree, not stable degree and so on. When we research the relation between the deformation and force, the real force is equal to bearing force, type (1) is the direct relation formula between the bearing capacity, force and material mechanical properties the three. Namely

$$
F_{\max }=\frac{T \times F}{s_{0}}=\frac{(1-E) F}{s_{0}}
$$

Where, $F_{\max }$ is the ultimate bearing capacity of the stratum; $T$ is the real degree of the formation; $F$ is the detection force; $E$ is the empty degree of the formation; $s_{0}$ is the area between the detection tool and the stratum. Style (3) is called the relationship formula among force, the ultimate bearing capacity and the nature of the strata.

\section{The Application Method of the New Formula}

Because the ultimate bearing capacity is equal to the limit value of the resistance, that is, because

$$
F_{\max }=-R_{\max }
$$

and the real force is equal to of the ultimate bearing capacity at the certain conditions, namely,

$$
F_{T}=F_{\max }
$$

in the test material mechanics properties, and may, according to the ratio between the resistance value and forces (i.e. detection force), obtain the real degree of the material (one of two the property parameter values of the material). That is,

$$
T=\frac{-R}{F}
$$

When force is less than the ultimate bearing capacity, the negative of the resistance is equal to the force, the material compaction is equal to 1 , namely, when $F<F_{\max }, R=-F, T=1$, this time the material do not deformation; As a force equal to limit bearing capacity, resistance to the negative force, the compaction of the material is also 1, which, at that time, $F=F_{\max }, R=-F, T=1$, the material do not deform; As a force more than limit bearing capacity, resistance less than force, the real degree of the material less than 1 , that is, when $F>F_{\max },-R<F, T<1$, the limit deformation is produced at this time. According to this law, we can research material mechanics properties, solve engineering problems, scientific prediction.

However, because in practice, the determination of the resistance value is very difficult, it is very difficult that using the aforementioned methods acquire the real degree $T$ value. In order to solve this problem, I have studied discussion the research project. Research shows that: the real degree $T$ is equal to 1 minus the false degree, rather the false degree is the amount that is obtained easy, so, the real degree $T$ is easy to get also. Namely, the real degree is equal to the rate between the deformation velocity of acting surface and the free falling speed of the role object. That is,

$$
\left\{\begin{array}{l}
E=\frac{v_{t}}{v_{0}}, \\
T=1-E .
\end{array}\right.
$$

Have type (7), we can calculate the real degree and limit bearing capacity directly, using the experimental data. For example, in the process of the foundation investigation, if the deformation force is 500 tons $/ \mathrm{m}^{2}$, the actual 
deformation amount (wedge formation depth) of the strata is $x_{T}=0.03$ meter (the deformation speed $v_{t}=u_{0}+a t=0.03 \mathrm{~m} / \mathrm{s}$ ), effect time $t=1$ second, the movement distance what the drilling tools should have a free drop is $x=v_{0} t+\frac{1}{2} a t^{2}=10 \mathrm{~m}$ (the free movement speed in under the gravity with is $v_{0}=u_{0}+g t=10 \mathrm{~m} / \mathrm{s}$ ), the contact area between drilling tools and formation is equal to $1 \mathrm{~m}^{2}$, so, the false degree of the strata is $E=0.03 / 10=0.003$, the real degree is $T=1-0.03 / 10=0.997$. Therefore, the ultimate bearing capacity of the formation is $F_{\max }=T \times F=0.997 \times 500=498.5$.

If the ultimate bearing capacity of the strata is more than $200 \mathrm{tons} / \mathrm{m}^{2}$, and the largest bearing capacity of meet the building requirements is 200 tons $/ \mathrm{m}^{2}$, the natural foundation can be used on the design, the contact area between the foundation and the building can be equal to $S_{0}$. If the practical bearing capacity of the formation is 150 tons $/ \mathrm{m}^{2}$, and the minimum bearing capacity of the building requirement is $200 \mathrm{tons} / \mathrm{m}^{2}$, then, the contact area between buildings and foundation must be $\frac{4}{3}$ time more than the area of natural foundation. That is, the type

$$
S \geq \frac{4}{3} S_{0}
$$

must be ensure. If the foundation is elongated, because length is certain, so, the problem of area is reduced to the width problems. That is, in the above conditions, the width designed of the foundation B must be equal to the $\frac{4}{3}$ times of the width of the natural foundation $\mathrm{B}_{0}$, or greater than. That is, must meet the requirements of the type

$$
B \geq \frac{4}{3} B_{0} .
$$

The problem on the deep Buried of foundation is another problem, this article does not discuss.

\section{The Invariance of the Ultimate Bearing Capacity}

The size of the ultimate bearing capacity depends on mechanical properties of materials. The material of specific material composition, specific physical structure, in certain circumstances, in particular action way and the size of the specific force, the ultimate bearing capacity is certain. But, the material mechanics properties changes with action and the size of the force and different environment changes. As a force less than or equal to limit bearing capacity, the material performance for entities, namely, the real degree of material is equal to 1 , the vanity degree is equal to zero. As a force more than limit bearing capacity, the material is represented but variants. At this time, the real degree of the materials is less than 1, the vanity degree is greater than zero. Generally speaking, the larger the force, the smaller the real degree of the deformation objects, the greater the false degree. The ultimate bearing capacity of the materials is to point to the maximum bearing capacity under ensuring what materials is not made condition, so, the ultimate bearing capacity of a material only a value, any numerical of less than the limit load are not limit bearing capacity.

In particular, the specific functions of the force, specific role by environment, material of ultimate bearing capacity is not change, namely, material of ultimate bearing capacity is one kind uncertainty value, not by the detection limit bearing capacity of the force and change size, the type $F_{\max }=T \times F / s_{0}$ is universal.

The nature what don't change limit bearing capacity with the size of the force to change is called the invariant property of the ultimate bearing capacity.

\section{The New Formula and the Traditional Formula Contras}

There are many parameters in the traditional formula that is used for computing the ultimate bearing capacity, not science, computing is very trouble. For example, Vesic formula

$$
f_{u}=\frac{1}{2} N_{r} \varsigma_{r} b \gamma+N_{q} \varsigma_{q} d \gamma_{0}+N_{c} \varsigma_{c} C_{k}
$$


involve three bearing capacity factors $\left(N_{r}, N_{q}, N_{c}\right)$, three basic shape correction coefficient $\left(\zeta r, \zeta_{q}, \zeta_{c}\right)$, involving basic length, width, and the internal friction Angle of soil $b$, buried depth $d(\mathrm{~m})$, and in the sticky gathered force standard $C_{k}(\mathrm{kPa})$ and so on, application up is not easy. But, the new formula (3) only involved a parameter, make problems greatly simplified. According to the type (3), as long as the soil to exert enough force in practice, make the soil in the deformation under the effect of overloading, can obtain the numerical of the limit bearing capacity and the parameters of the nature of the soil.

For example, if the soil is acted by the standard penetration, detection force is provided by standard penetration hammer, then, the initial momentum of the standard penetration is $I_{0}=M g t_{0} ; M$ is the quality of standard penetration hammer; $g$ is gravity acceleration; $t_{0}=\sqrt{\frac{2}{g}} ; I_{0}=M \sqrt{2 g}$; the action time of standard penetration to the strata is $t$ (the time of the strata deformation); $t=0.1$ seconds (the weaker strata, its deformation more time); the amount of the wedging deformation is $x_{T}=0.03$ meters; suppose no strata block, and standard penetration through a free drop, and, $\mathrm{g}=10 \mathrm{~m} / \mathrm{second}$, then, the distance of the standard penetration through a free drop should be $x=v_{0} t+\frac{1}{2} g t^{2}=0.478$ meters, and so, according to type (7): the waste degree of the strata is $E=\frac{0.03}{0.478} \approx 0.0627215$, the real degree is $T=1-E=1-0.0627215=0.9372785$.

The ultimate bearing capacity of $t$ strata is

$$
\begin{aligned}
& N_{\max }=T F / s_{0}=0.9372785 M v_{0} / s_{0} \\
& =0.9372785 \times 63.5 \times 10 / s_{0} \\
& =\frac{595.1718475}{s_{0}}\left(\mathrm{~kg} / \mathrm{m}^{2}\right)
\end{aligned}
$$

In this type, $\mathrm{s}_{0}$ is equal to the area between standard penetration and strata.

Visible, and Vesic formula or other the traditional formula compared, the new formula (3) has great advantages.

\section{The Traditional Load Test, the Bearing Capacity of In Situ Test Values}

At present, the load test is considered the most accurate, the most reliable foundation bearing capacity test method. This method is: to the formation for loading, let stratum deformation, then determine the bearing capacity of the strata. If stratum didn't produce deformation, didn't achieve ultimate bearing capacity; If produce tiny stratum deformation, exceed limit bearing capacity. What the values of the ultimate bearing capacity is between not deformation and deformation, no exact formula. With no definite theory formula, so, the value of the ultimate bearing capacity that is conservatively confirmed for large data, and do not give accurate data. Usually the maximum capacity with engineering requirements to pressure, when pressure, a tool to detect motionless, what it is considered just meet the requirements, the values are not definite. To be able to check data,

The way of in situ test is mainly standard penetration and power detection. New regulations before, the mutual control data the hammer number of the standard penetration test and the bearing capacity of the sand or soil layer in table can be checked.The following excerpt part:

1) when the strike is 30 more than, the close-grained gravel sand, coarse sand, medium sand (porosity were less than 0.60 ) corresponding bearing capacity is 40 tons $/ \mathrm{metre}^{2}$;

2) when hit number less than or equal to 30 and greater than 15 , medium dense (porosity are greater than 0.60 and less than 0.75 ) gravel sand, coarse sand, medium sand corresponding allow bearing capacity for 30 tons/metre ${ }^{2}$, (porosity are greater than 0.70 and less than 0.85 ) fine sand, powder sand corresponding allow bearing capacity of $15-20$ tons/ metre';

3) when hit number less than or equal to 15 and is equal to or greater than 10 , a little secret (porosity are greater than 0.75 and less than 0.85 ) of the gravel sand, sand, medium sand corresponding allow bearing capacity for 20 tons $/$ metre $^{2}$, (porosity are greater than 0.85 and less than 0.95 ) of the fine sand, powder sand corresponding allow bearing capacity of $10-15$ tons/ metre $^{2}$.

4) The allow bearing capacity of the old clay and general cohesive soil, when the hammer the number three, respectively $5,7,9,11,13,15,17$ and $19,21,23$, is its corresponding allow bearing capacity for 12 and 16 respectively, 20, and $24,28,32,36,42,50,58,66$ tons/ metre $^{2}$. 
These data is not the data of the bearing capacity and standard penetration the corresponding, just some of the number determined by some people, and the comparable data of the inaccurate limit bearing capacity, but these ultimate bearing capacity than the actual big many, the data was used without problems. Therefore, in situ test, load experiment data is not exactly.

\section{Conclusion}

Through the above discussion that:

First, there are unscientific problems in the current bearing capacity theory, the calculation formula of the bearing capacity, the detection methods.

Second, there is the direct relation between the ultimate bearing capacity and forces. In the engineering survey research work, this kind of relation rule can be used. The bearing capacity of the foundation can directly be calculated.

Three, the current engineering mechanics calculation and engineering geology research method needs to be improved.

In addition, must be mentioned, this paper mentioned calculation methods and formulas need to measure time, given to the actual research increased difficulties and workload. Traditional method does not measure time, no time variable, but not be able to get the accurate data. Therefore, the study also waits for a great deal of experiments.

\section{References}

Danziger, F. A. B., Almeida, M. S. S. \& Sills, G. C. (1997). The significance of the strain path analysis in the inter pretation of piezocone dissipation data. Geotechnique, 47(5), 901-914. http://dx.doi.org/10.1680/geot.1997.47.5.901

Lunne, T., Robertson, P. K. \& Powell, J. J. M. (1997). Cone penetration Testing in Geotechnical Practice. Chapman \& Hall.

Schnaid, F., Houlsby, G. T. (1991). An assessment of chamber size effects in the calibration of insitutest in sand. Geotechnique, 41(3), 437-455. http://dx.doi.org/10.1680/geot.1991.41.3.437

Teh, C. \& Houlsby, G. (1991). An analytical study of the cone pentration test in clay. Geotechnique, 41(1), 17-34. http://dx.doi.org/10.1680/geot.1991.41.1.17

Wang Chang Yi \& He Ke Qiang. (2009). The law of the unity of opposites is applied in landslip research. Journal of Qingdao Technological University, 3.

Wang Chang Yi \& He Ke Qiang. (2010). Concerning the groundwater movement rules and its research methods. Journal of Qingdao Technological University, 35.

Wang Chang Yi \& Sun Jie. (2010). The outline of the action science. The urban construction, 3, 315.

Wang Chang Yi \& Sun Jie. (2010). The principle of the action science of landslide forecast role theory. The Urban Construction, 2, 183.

Wang Chang Yi. (2010). On mechanical defects. Humanities \& Social Sciences Forum, 9, 59.

Zhang Xi Fa. (1988). Engineering geology in situ test. Beijing: Geological Publishing House. 